\title{
Molecular determinants of brown adipocyte formation and function
}

\author{
Stephen R. Farmer ${ }^{1}$ \\ Department of Biochemistry, Boston University School of Medicine, Boston, Massachusetts 02118, USA
}

Humans contain essentially two types of adipose tissue: brown adipose tissue (BAT) and white adipose tissue (WAT). The function of WAT is to store fat while that of BAT is to burn fat for heat production. A potential strategy to combat obesity and its related disorders is to induce the conversion of WAT into BAT. In this issue of Genes \& Development, Kajimura and colleagues (pp. 1397-1409) have identified a mechanism by which PRDM16, the principal regulator of brown adipocyte formation and function, can simultaneously induce BAT gene expression, while suppressing WAT gene expression. The studies suggest that PRDM16 and its associated coregulators PPAR $\gamma$ coactivator-1 $\alpha$ (PGC-1 $\alpha)$ and C-terminal-binding protein $1 / 2$ (CtBP1/2), which control the switch from WAT to BAT, are potential targets for development of obesity-related therapeutics.

The modern world is in the midst of an obesity epidemic that is growing to the extent that, in 2003-2004, 32\% of US adults were obese and $>50 \%$ were considered overweight (Ogden et al. 2006). This increase in adiposity has led to a significant increase in obesity-related disorders including type 2 diabetes, cardiovascular disease, hypertension, and certain cancers (Li et al. 2005). Humans contain essentially two types of adipose tissue: brown adipose tissue (BAT) and white adipose tissue (WAT). The most predominant by far is WAT, which functions to store energy in the form of triglyceride-containing intracellular droplets as well as to secrete a host of hormones and cytokines (adipokines) that regulate overall energy balance by affecting the function of other tissues, most notably the brain, muscle, and liver (Gesta et al. 2007). The principal function of BAT is to burn fat to generate heat, particularly in newborns as a protective measure during the initial hours following birth into a cold environment. BAT depots decrease significantly in size as humans mature, existing in adults within small, defined locations throughout the body as well as distributed as small pockets within WAT depots (Cannon and Nedergaard 2004). Obesity is primarily associated with an ex-

[Keywords: PRDM16; adipogenesis; CtBP; PGC-1; PPAR- $\gamma$; resistin] ${ }^{1}$ Correspondence.

E-MAIL farmer@biochem.bumc.bu.edu; FAX (617) 638-5339.

Article is online at http://www.genesdev.org/cgi/doi/10.1101/gad.1681308. pansion of the WAT depots that exist under the skin (subcutaneous) and around our internal organs (visceral). The extensive increase in individual white fat cell size (due to accumulation of triglycerides) in obese individuals results in a perturbation of function, including changes in hormone and cytokine secretion that lead to a disruption in normal metabolic homeostasis and eventual associated pathologies such as insulin resistance and type 2 diabetes. The fact that BAT burns triglycerides (thermogenesis) and also secretes fewer cytokines offers a potential strategy to combat obesity by developing drugs that can enhance brown fat formation or induce the transdifferentiation of WAT into BAT. Development of such therapeutics, however, requires significant knowledge of the molecular mechanisms controlling the formation and function of brown fat cells. In this issue of Genes \& Development, Kajimura et al. (2008) demonstrate that PRDM16, a zinc-finger protein selectively expressed in BAT, controls brown adipocyte formation by inducing expression of BAT genes and simultaneously suppressing expression of select WAT genes. This switching mechanism appears to be due to the ability of PRDM16 to interact with a known repressor, C-terminal-binding protein-1 (CtBP-1) and CtBP-2, to inhibit the WAT genes, or with the thermogenic transcription factor PPAR $\gamma$ coactivator- $1 \alpha$ (PGC- $1 \alpha$ ) to stimulate brown gene expression.

\section{Function of BAT}

BAT exists within small mammals in distinct locations being innervated by the sympathetic nervous system (SNS) as well as supplied by the circulation through a dense microvasculature (Cannon and Nedergaard 2004). Upon cold exposure, heat production in the animals is facilitated by the SNS-stimulated release of catecholamines, which activates thermogenesis and dissipation of the generated heat through the circulation. This heat is generated by BAT through accelerated oxidation of stored lipids within brown adipocytes, facilitated by large numbers of mitochondria. Additionally, brown adipose cells express uncoupling protein-1 (UCP-1), a proton transporter that uncouples electron transport from ATP production, allowing the energy to dissipate as heat. UCP-1 is exclusively expressed in brown adipocytes, and while analysis of WAT depots displays some low level of 
UCP-1, it is likely due to the sparse distribution of brown fat cells throughout the tissue. Brown adipocytes also produce many other proteins that are modestly produced in white cells, including mitochondrial proteins involved in lipid oxidation as well as electron transport. Interestingly, brown cells also produce significantly lower amounts of certain hormones and cytokines that are abundantly produced in white cells. These include proteins that are associated with obesity-related pathologies, most notably resistin, which contributes to insulin resistance (Steppan et al. 2001), and angiotensinogenin, which contributes to hypertension (Engeli et al. 2000). As mentioned above, BAT is highly vascularized, containing many small capillaries that weave through the mass of individual brown adipocytes. In contrast, WAT has significantly fewer blood vessels; furthermore, as WAT becomes increasingly enlarged during obesity it also becomes less vascularized (Goossens 2007). It is very likely that the dense vascular bed within BAT is the result of a correspondingly large production of angiogenic factors such as VEGF by the brown adipocytes. As mentioned, BAT functions to keep small animals warm in cold environments, including not only mice but also newborn humans; consequently, small mammals contain significant quantities of brown fat cells. In humans, however, BAT decreases shortly after birth and was, until recently, considered to have an insignificant function in adults. Unexpected evidence has arisen, however, for the presence of active BAT in adult humans from the use of fluorodeoxyglucose positron emission tomography (FDG PET) to detect tumors (Truong et al. 2004). Such PET scans have located hypermetabolic BAT in the cervical, supraclavicular, paravertebral, mediastinal, para-aortic, and suprarenal regions (but no interscapular) of adult subjects (Nedergaard et al. 2007). This discovery, therefore, reintroduces the notion that BAT contributes to energy balance in humans and might be a therapeutic target in the fight to combat obesity-associated disorders.

\section{Formation of adipose tissue}

Adipose tissue is generally considered to arise from a mesodermal origin along with muscle, cartilage, and bone, but the precise mesenchymal stem cell lineages that produce white versus brown adipocytes are not known. It has been assumed, though, that the mesenchyme produces a population of common progenitors that develop into either white or brown adipocytes in response to tissue-specific effectors. The evolutionary and developmental features of BAT and WAT suggest that they are quite distinct tissues with separate origins. BAT evolved significantly later than WAT, in parallel with the evolution of nonshivering thermogenesis and endothermo-regulation in birds through to mammals, whereas fat is stored within some form of WAT depot in fish and amphibians that lack BAT (Gesta et al. 2007). BAT emerges during fetal development significantly earlier than WAT, reaching its maximum size at about birth, whereas development of WAT begins at midgesta- tion (humans) or shortly after birth (rodents) and gradually increases in size throughout life. In fact, some investigators are now considering the notion that brown adipocytes arise from a separate and distinct population of progenitors. Timmons et al. (2007) have reported recently that brown cells possess a "myogenic" signature, suggesting that they share a common mesenchymal origin with muscle, a tissue that also burns lipids for energy production. Additionally, recent unpublished lineage-tracking studies by Spiegelman and colleagues (B. Spiegelman, pers. comm.) using the myf-5 promoter also suggest that brown adipocytes have a myogenic origin. The differentiation of brown and white preadipocytes into mature fat cells, however, appears to employ a similar canonical transcriptional pathway of nuclear factors that regulates production of proteins and processes that are common to both cell types, including lipogenesis and insulin-dependent glucose transport. Functions that are unique to each tissue are likely regulated by cell type-specific factors, as will be discussed in more detail below. Many studies during the last decade have identified a cascade of adipogenic factors that regulate adipogenesis in both white and brown cells (Farmer 2006; Rosen and MacDougald 2006; Gesta et al. 2007). The most notable are PPAR $\gamma$ and $\mathrm{C} / \mathrm{EBP} \alpha$, which are generally considered the master regulators of the process, with PPAR $\gamma$ being indispensable for white and brown adipocyte formation. The absence of $\mathrm{C} / \mathrm{EBP} \alpha$ in mice prevents the development of WAT but has limited effect on BAT development. The function of PPAR $\gamma$ is to induce expression of many hundreds of target genes involved in lipid and glucose metabolism, including mitochondrial biogenesis and secretion of adipokines. C/EBP $\alpha$ functions to maintain $\operatorname{PPAR} \gamma$ production as well as regulate subsets of adipocyte genes that regulate processes that include adiponectin secretion and insulin-dependent glucose uptake.

\section{Nuclear factors regulating brown versus white adipogenesis}

Several nuclear factors have been associated with the formation of brown fat cells (Gesta et al. 2007). Until the recent discovery of PRDM16, the most notable was PGC-1 $\alpha$ (Puigserver et al. 1998). PGC-1 $\alpha$ is highly expressed in BAT compared with WAT and is responsible for regulating mitochondrial biogenesis and thermogenesis. Other studies show that it functions in several tissues, including brain, liver, skeletal muscle, and heart, as well as BAT, as a global regulator of energy balance through its coactivation of a diverse group of transcription factors (Houten and Auwerx 2004; Lin et al. 2004, 2005). Mice deficient in PGC-1 $\alpha$ are cold-sensitive with low expression of UCP-1 and a morphologically abnormal BAT (Lin et al. 2004). Loss of PGC-1 $\alpha$ in brown fat cells in culture, however, does not alter brown adipogenesis but severely reduces the induction of thermogenic genes by cAMP. Interestingly, loss of both PGC- $1 \alpha$ and PGC-1 $\beta$ from brown preadipocytes in culture prevents induction of the brown features, including increased 
mitochondria production and function without affecting adipogenesis (Uldry et al. 2006). It is generally accepted, therefore, that PGC- $1 \alpha$ is the critical regulator of adaptive thermogenesis in responsive tissues, but it is not the master regulator of brown adipocyte formation.

\section{PRDM16: a master regulator of brown adipocyte formation}

To identify the principal factor(s) regulating commitment of progenitors to the brown lineage, Spiegelman and colleagues (Seale et al. 2007) employed global expression analysis of murine transcriptional components using white and brown tissue RNA samples from C57BL/6 mice. The screen identified three genes-Lhx8, Zic1, and PRDM16 - that ultimately met their criteria of being preferentially expressed in brown versus white fat. PRDM16 is a $140-\mathrm{kDa}$ zinc-finger PR (PRD1-BF1-RIZ1 homologous) domain-containing protein that induces a program of gene expression as well as mitochondrial biogenesis/oxygen consumption, consistent with the brown phenotype, when ectopically expressed in white preadipocytes in culture or white depots in the animal (Seale et al. 2007). Furthermore, knockdown of PRDM16 in brown fat cells ablates their brown characteristics. PRDM16 appears to function through its ability to coactivate both PGC-1 $\alpha$ and PGC-1 $1 \beta$. In this issue of Genes \& Development, Kajimura et al. (2008) extend this initial identification of PRDM16 as a regulator of brown adipocyte formation to define the molecular mechanisms by which it can simultaneously induce brown genes while suppressing white genes. The data show that PRDM16 can interact with either PGC- $1 \alpha / \beta$ or CtBPs to activate brown genes or to suppress white gene expression, respectively. The binding of each of these nuclear factors to PRDM16 is mutually exclusive even though they associate with distinct domains. In fact, CtBPs bind to a PLDLS motif at position 804-808 within PRDM16, and mutation of this site prevents the suppression of white genes in brown cells but has no effect on the induction of brown genes. The mechanisms regulating the switch in binding of these factors to PRDM16 is not known. Additional data by Kajimura et al. (2008) suggest that PRDM16 docks on specific transcription factors associated with the promoters/enhancers of the respective target genes and that this association might then determine whether PGC- $1 \alpha / \beta$ or CtBPs initiates the formation of either a coactivator or corepressor complex. The precise transcription factor(s) directing PRDM16 to the respective target genes is also not known. It is possible that multiple factors are involved. In the case of the brown genes, likely candidates include PPAR $\gamma$, whereas for the white genes a recent study by Lazar and colleagues (Hartman et al. 2002) has positioned the binding of $\mathrm{C} / \mathrm{EBP} \alpha$ to the same region of the resistin promoter through which PRDM16/CtBP appears to impose its repressive function.

The need for a brown-specific determinant such as PRDM16 to induce expression of genes associated with mitochondrial biogenesis, oxidative phosphorylation, and oxidation of lipids is obvious when considering the function of BAT. However, a requirement to suppress expression of WAT genes coding for secreted cytokines and hormones is not so apparent. It is possible that due to the relatively small mass of BAT, evolution has selected the expansive WAT system to fulfill the duties of supplying the organism with the necessary adipokines such as resistin and angiotensinogen. It is also possible that these secreted substances counteract some unknown critical function(s) of BAT, such as adaptive thermogenesis or angiogenesis; consequently, these white genes need to be silenced to facilitate this activity.

\section{Role of PPAR $\gamma$ in the switch from white to brown phenotype}

As discussed above, PPAR $\gamma$ functions to regulate the underlying adipogenic programs common to both white and brown adipocytes and is, without question, an indispensable component of the machinery regulating formation of each cell type. It is also possible that its activity can favor one phenotype over the other. Several recent studies have shown that exposure of white adipocytes either in culture or in animals to potent PPAR $\gamma$ ligands such as rosiglitazone induces a "browning" of the white cells, as characterized by an increase in mitochondrial mass and structure as well as a markedly enhanced oxygen consumption and lipid oxidation (Wilson-Fritch et al. 2003, 2004). This process is likely due to a PPAR $\gamma$ ligand-associated induction of mitochondrial genes, including UCP-1 and cytochrome c oxidase (Cox), subunit VIIIb (Cox8b), and subunit VIIa1 (Cox7a1) (Wilson-Fritch et al. 2004). Additionally, other studies have documented an extensive inhibition of adipokine production including resistin, $\alpha 1$-acidglycoprotein, and haptoglobin by treatment of white adipocytes with thiazolidinedione (TZD) and non-TZD synthetic PPAR $\gamma$ ligands (Steppan et al. 2001; do Nascimento et al. 2004; Castriota et al. 2007). It is intriguing that supplementing with an additional PPAR $\gamma$ ligand would elicit such a striking effect on gene expression under conditions where PPAR $\gamma$ activity (and endogenous ligand production) is already high. To this end, the synthetic ligand (TZD) must somehow be stimulating PPAR $y$ to perform a set of brownspecific functions in white cells that endogenous ligands are incapable of activating. This notion raises the possibility that brown adipocytes produce a unique set of PPAR $\gamma$ ligands that differ functionally from those produced in white cells. The most likely mechanism responsible for the browning effects of "superactive" PPAR $y$ in white cells is its induction of PRDM16 complexed with PGC- $1 \alpha / \beta$ or CtBPs. In fact, white adipocytes already express PGC-1 $\beta$ and CtBPs, and recent studies show an extensive induction of PGC- $1 \alpha$ in WAT in response to TZDs (Wilson-Fritch et al. 2004). There is no information on whether superactivation of PPAR $\gamma$ by TZDs or other ligands is capable of inducing PRDM16 in white adipocytes.

It is very likely that identification of the molecular mechanisms regulating PRDM16 gene expression will 
provide great insight into the early determinants of brown and white cell lineages during development. Such information might also provide additional targets for the development of potential therapeutics for treatment of obesity-related disorders. Possible candidates for upstream regulators of PRDM16 gene expression include factors involved in the commitment of mesenchymal stem cells to the myogenic lineage, most notably myf5 (B. Spiegelman, pers. comm.). It is also possible that PPAR $\gamma$ associating with a potent ligand produced in brown cells is capable of forming complexes that bind to and activate the PRDM16 gene. PRDM16 through association with PGC-1 $\beta$ would then be capable of inducing PGC- $1 \alpha$ expression via a feedback mechanism to generate the machinery necessary to regulate the brown phenotype. A component of this machinery is, of course, a set of repressor complexes containing CtBPs, which will suppress white genes such as resistin, but could also suppress those factors maintaining white gene expression such as receptor-interacting protein 140 (RIP140) (see below).

\section{Transcriptional repressors as important regulators} of the brown phenotype

CtBP-1 and CtBP-2 interact as dimers with a variety of sequence-specific DNA-binding transcriptional repressors to form complexes that can also recruit histonemodifying enzymes as well as the small ubiquitin-related modifier (SUMO)-conjugating E2 enzyme UBC9 and a SUMO E3 ligase (HPC2) (Chinnadurai 2007). Repression of transcription likely involves these modifications as well as the ability of CtBPs to antagonize the activity of the global transcriptional coactivators p300/ CBP. It is interesting that the CtBPs exhibit structural and functional similarity to D-isomer-specific 2-hydroxy acid dehydrogenases (D2-HDH), although no specific role for dehydrogenase activity, shown to occur in in vitro assays, has been demonstrated to participate in transcriptional repression in vivo. There does appear to be a role for $\mathrm{NAD}(\mathrm{H})$ dinucleotides in regulating the activity of CtBPs by stimulating dimerization as well as enhancing interaction of CtBPs with PLDLS-containing target proteins (Kumar et al. 2002; Zhang et al. 2002). In fact, interaction of CtBPs with repressors appears to be stimulated in the presence of increased $\mathrm{NADH} / \mathrm{NAD}^{+}$ ratio, suggesting that CtBP might be a redox sensor. Consistent with this notion, there are data showing that hypoxia, which should increase the $\mathrm{NADH} / \mathrm{NAD}^{+}$ratio, enhances the CtBP-dependent repression of E-cadherin during tumor cell migration (Zhang et al. 2006). Additional data suggest that the ability of $\mathrm{HIC1}$ : $\mathrm{CtBP}$ complexes to regulate SIRT1 expression is also responsive to the $\mathrm{NADH} / \mathrm{NAD}^{+}$ratio (Zhang et al. 2007). It will be interesting to determine whether the PRDM16/CtBP-associated repression of white genes in brown cells is similarly responsive to the redox state of the cell.

In reviewing specific repression processes regulating brown versus white fat development and function, it is also important to discuss the role of RIP140, since it has also been proposed to be a global regulator of genes that control mitochondrial pathways and energy balance (Christian et al. 2006; Parker et al. 2006). RIP140 is a ligand-dependent repressor of the transcriptional activity of nuclear receptors (NR) including estrogen receptor (ER) and PPARs. Other repressors of NRs such as SMRT and NCoR dissociate from the receptor in response to binding of the ligand. RIP140-null mice are lean due to a $70 \%$ reduction in total body fat present in the WAT depots but with the same number of smaller adipocytes relative to controls and no change in food consumption (Leonardsson et al. 2004). They are also resistant to a high-fat-diet-induced obesity and are protected from ageinduced as well as diet-induced hepatic steatosis. These observations suggest that the null mice oxidize the consumed fat rather than storing it. Consistent with this notion, other studies have shown that suppression of RIP140 in white adipocytes by siRNA technology leads to a significantly enhanced expression of BAT genes that are targets of PGC-1 $\alpha$ (Powelka et al. 2006). It appears, therefore, that RIP140, which is highly expressed in white cells, functions to suppress the brown phenotype. It is relevant that a component of the repressive activity of RIP140 is CtBP (Christian et al. 2006). Specifically, RIP140 is composed of four repression domains, termed RD1-RD4, that act as platforms for the formation of corepressor complexes. The mechanism for repression by RD1 and RD2 involves recruitment of histone deacetylaces (HDACs) that depends on association with CtBPs. In an attempt to understand the molecular mechanisms regulating the switch from a brown to white phenotype, a model is presented (Fig. 1) in which various extracellular effectors that modulate redox state such as hypoxia and nutrients might control expression of coactivators (PGC- $1 \alpha / \beta)$ and/or repressors (CtBPs) that can associate with specific platform proteins (PRDM16 or RIP140) to produce coactivator or corepressor complexes docked on specific target genes. It is likely that CtBPs are involved in repression of both white and brown genes.

\section{Other important functional differences between brown and white adipocytes: angiogenesis}

BAT is highly vascularized compared with WAT, presumably because it needs a copious blood supply to provide oxygen and nutrients, primarily lipids, for heat production and to ensure a rapid redistribution of the heat to the organism (Cannon and Nedergaard 2004). In the case of WAT, there are several studies showing a decrease in blood flow and vascular network with obesity, which leads to local adipose hypoxia and extensive increases in lactate concentrations (for review, see Goossens 2007). Such effects have been shown to contribute to ER stress within the adipocytes, leading to perturbations in adiponectin secretion (Ye et al. 2007). It appears, therefore, that the hypoxic WAT is incapable of inducing angiogenesis to levels that can compensate for the reduced blood flow and low oxygen/nutrients. The master regulator of the hypoxia response, hypoxia-induced factor $1 \alpha$ (HIF$1 \alpha$ ), is induced during obesity in WAT (Cancello et al. 


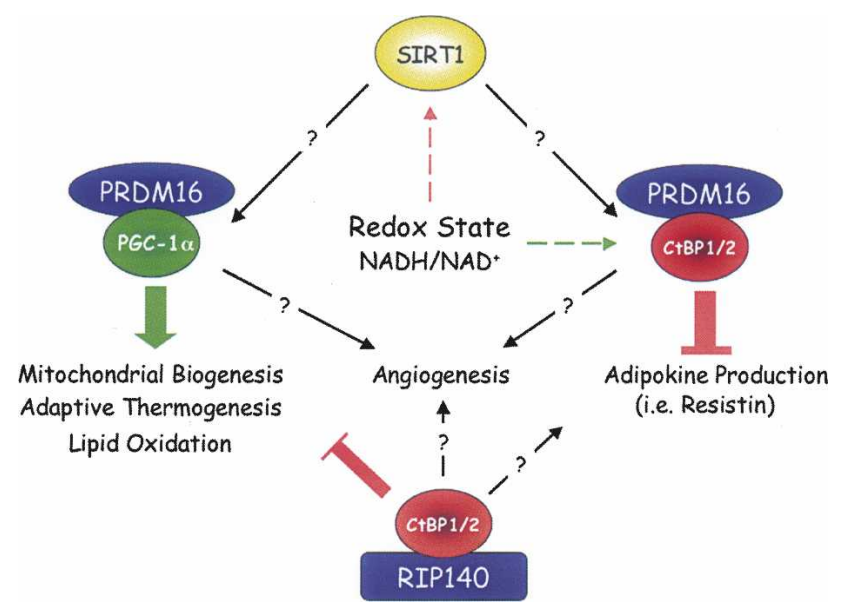

Figure 1. Mechanisms controlling the switch from white to brown phenotype. Studies by Kajimura et al. (2008) demonstrate that PRDM16 can interact with either PGC- $1 \alpha / \beta$ or CtBPs to activate brown genes or to suppress white gene expression, respectively. Studies by Parker and colleagues (Christian et al. 2006; Parker et al. 2006; Powelka et al. 2006) have also shown that RIP140 in association with CtBPs can suppress mitochondrial gene expression in white adipocytes. It will be interesting to determine additional functions for PRDM16 and RIP140 coregulator complexes in controlling other features of the brown versus white fat cell phenotype including angiogenesis and adipokine production. Furthermore, knowledge of the involvement of changes in redox state (i.e., hypoxia and nutrients) in controlling these processes will be very informative for the development of therapeutics for obesity-associated disorders.

2005). However, increased HIF-1 $\alpha$ protein expression is incapable of inducing an angiogenic response, potentially due to decreased activity or absence of a crucial factor. Recent studies by Spiegelman and colleagues (Arany et al. 2008a) have provided some insight into why BAT is significantly more vascularized than WAT and why WAT cannot compensate for the reduced blood flow during obesity. Arany et al. (2008a) demonstrate that PGC- $1 \alpha$ is induced by a lack of nutrients and oxygen and stimulates VEGF expression and angiogenesis in cultured muscle cells and brown adipocytes and skeletal muscle in vivo. Interestingly, the induction of angiogenesis by PGC- $1 \alpha$ does not require HIF-1, but instead involves a coactivation of the orphan nuclear receptor ERR- $\alpha$ (estrogen-related receptor- $\alpha$ ) on the VEGF promoter. In light of this information, it is interesting to consider whether components of the machinery regulating brown adipocyte function as discussed above are also involved in mediating the response of BAT to hypoxia and/or nutrients. The data also suggest potential therapeutic strategies to enhance blood flow in obese WAT, most notably by increasing expression of PGC- $1 \alpha$ in white adipocytes (see below). The mechanism(s) responsible for the induction of PGC- $1 \alpha$ in response to hypoxia or reduced nutrients is not investigated by Arany et al. (2008a). It is well known that hypoxia activates HIF-1 $\alpha$ in most other hypoxic tissues/cells through an elaborate process involving the $\mathrm{O}_{2}$-dependent hydroxylation of
HIF- $1 \alpha$ by a series of hydroxylases (egln 1 , egln 2 , and egln3) that target it for degradation in the proteasome; low $\mathrm{O}_{2}$ tension, therefore, leads to reduced hydroxylation and accumulation/activation of HIF-1 $\alpha$ followed by induction of its target genes, including VEGF and other angiogenic factors (Semenza 2001, 2007a,b). It is unlikely, though, that a similar mechanism is involved in hypoxia-associated activation of PGC-1 $\alpha$. There are alternative mechanisms, however, based on discussions above, by which low oxygen and/or nutrients might initiate such a PGC-1 $\alpha$-dependent angiogenic process (Fig. 1). Hypoxia can lead to an increase in intracellular NADH levels in both the cytoplasmic (NADHc) as well as mitochondrial (NADHm) compartments, thereby raising the $\mathrm{NADH} / \mathrm{NAD}^{+}$ratio (Nyengaard et al. 2004). As discussed earlier, NADH enhances the binding of CtBPs to repressor complexes; consequently, PGC- $1 \alpha$ activity could be induced during hypoxia through a NADH-associated repression of factors that inhibit PGC-1 $\alpha$ (i.e., RIP140). NADH/NAD ${ }^{+}$also has the ability to influence the activity of the $\mathrm{NAD}^{+}$-dependent deacetylase, SIRT1, which has been shown to deacetylate PGC- $1 \alpha$ and in doing so affects its transcriptional activity in both a positive as well as negative manner (Rodgers et al. 2005). Finally, it will be very informative to determine whether PRDM16 is involved in regulating the response of metabolic tissues such as muscle and BAT to low oxygen and nutrients.

\section{TZDs and therapeutic strategies to enhance brown fat production}

The data discussed above introduce several targets that can be considered for the development of therapeutics to enhance the brown phenotype within WAT, which effectively should lead to a reduction in obesity-associated disorders. Without question some of the most effective drugs to combat obesity-related insulin resistance and type 2 diabetes are the TZD family of insulin sensitizers and potent ligands for PPAR $\gamma$. As discussed, several studies have shown that exposure of obese WAT to TZDs causes a browning of the white adipocytes that includes not only induction of mitochondrial biogenesis and enhanced oxygen consumption but also a suppression of resistin and other white-selective genes. Unfortunately, the use of TZDs has met with much skepticism due to potentially life-threatening cardiac side affects as well as an increase in adipose mass. What is needed is the development of potent PPAR $\gamma$ ligands that only induce the brown phenotype without enhancing white adipose features (i.e., fat accumulation) or negative effects on cardiac tissue. One strategy is to screen for small molecules that selectively activate PPAR $\gamma$ to induce mitochondrial gene expression without affecting lipogenic genes. Recent investigations have already targeted SIRT1 by treating diabetic mice with resveratrol and other synthetic activators of the deacetylase, resulting in improvements in insulin sensitivity and mitochondrial capacity, likely through the activation of PGC- $1 \alpha$ in skeletal muscle (Baur et al. 2006; Lagouge et al. 2006; Milne et al. 2007). 
In this regard, Arany et al. (2008b) performed a highthroughput screen to identify additional small molecules that induce PGC- $1 \alpha$ in muscle and discovered that microtubule inhibitors as well as protein synthesis inhibitors potently enhance activity of the coactivator, resulting in expression of mitochondrial genes and enhanced oxygen consumption (Arany et al. 2008b).

In summary, the studies discussed above raise many questions concerning the mechanisms controlling adipose tissue formation and function, as illustrated in Figure 1. While the studies from Kajimura et al. (2008) and Seale et al. (2007) identify PRDM16 as a likely master regulator of brown adipocyte differentiation, the next crucial step will be to determine the factors responsible for promoting PRDM16 expression. Such factors will certainly provide significant insight into the stem cell origins that lead to brown fat formation and will likely act as targets for development of obesity-associated therapeutics.

\section{Acknowledgments}

I thank Drs. Kathryn E. Davis and Cecile Vernochet for critical review of the manuscript and constructive discussions. I particularly thank Kathryn for designing the figure. I also acknowledge support from the National Institutes of Health.

\section{References}

Arany, Z., Foo, S.Y., Ma, Y., Ruas, J.L., Bommi-Reddy, A., Girnun, G., Cooper, M., Laznik, D., Chinsomboon, J., Rangwala, S.M., et al. 2008a. HIF-independent regulation of VEGF and angiogenesis by the transcriptional coactivator PGC- $1 \alpha$. Nature 451: 1008-1012.

Arany, Z., Wagner, B.K., Ma, Y., Chinsomboon, J., Laznik, D., and Spiegelman, B.M. 2008b. Gene expression-based screening identifies microtubule inhibitors as inducers of PGC- $1 \alpha$ and oxidative phosphorylation. Proc. Natl. Acad. Sci. 105: 4721-4726.

Baur, J.A., Pearson, K.J., Price, N.L., Jamieson, H.A., Lerin, C., Kalra, A., Prabhu, V.V., Allard, J.S., Lopez-Lluch, G., Lewis, K., et al. 2006. Resveratrol improves health and survival of mice on a high-calorie diet. Nature 444: 337-342.

Cancello, R., Henegar, C., Viguerie, N., Taleb, S., Poitou, C., Rouault, C., Coupaye, M., Pelloux, V., Hugol, D., Bouillot, J.L., et al. 2005. Reduction of macrophage infiltration and chemoattractant gene expression changes in white adipose tissue of morbidly obese subjects after surgery-induced weight loss. Diabetes 54: 2277-2286.

Cannon, B. and Nedergaard, J. 2004. Brown adipose tissue: Function and physiological significance. Physiol. Rev. 84: 277359.

Castriota, G., Thompson, G.M., Lin, Y., Scherer, P.E., Moller, D.E., and Berger, J.P. 2007. Peroxisome proliferator-activated receptor $\gamma$ agonists inhibit adipocyte expression of $\alpha 1$-acid glycoprotein. Cell Biol. Int. 31: 586-591.

Chinnadurai, G. 2007. Transcriptional regulation by C-terminal binding proteins. Int. J. Biochem. Cell Biol. 39: 1593-1607.

Christian, M., White, R., and Parker, M.G. 2006. Metabolic regulation by the nuclear receptor corepressor RIP140. Trends Endocrinol. Metab. 17: 243-250.

do Nascimento, C.O., Hunter, L., and Trayhurn, P. 2004. Regulation of haptoglobin gene expression in 3T3-L1 adipocytes by cytokines, catecholamines, and PPAR $\gamma$. Biochem. Biophys. Res. Commun. 313: 702-708.

Engeli, S., Negrel, R., and Sharma, A.M. 2000. Physiology and pathophysiology of the adipose tissue renin-angiotensin system. Hypertension 35: 1270-1277.

Farmer, S.R. 2006. Transcriptional control of adipocyte formation. Cell Metab. 4: 263-273.

Gesta, S., Tseng, Y.H., and Kahn, C.R. 2007. Developmental origin of fat: Tracking obesity to its source. Cell 131: 242256.

Goossens, G.H. 2007. The role of adipose tissue dysfunction in the pathogenesis of obesity-related insulin resistance. Physiol. Behav. doi: 10.1016/j.physbeh.2007.10.010.

Hartman, H.B., Hu, X., Tyler, K.X., Dalal, C.K., and Lazar, M.A. 2002. Mechanisms regulating adipocyte expression of resistin. J. Biol. Chem. 277: 19754-19761.

Houten, S.M. and Auwerx, J. 2004. PGC-1 $\alpha$ : Turbocharging mitochondria. Cell 119: 5-7.

Kajimura, S., Seale, P., Tomaru, T., Erdjument-Bromage, H., Cooper, M.P., Ruas, J.L., Chin, S., Tempst, P., Lazar, M.A., and Spiegelman, B.M. 2008. Regulation of the Brown and white fat gene programs through a PRDM16/CtBP transcriptional complex. Genes \& Dev. (this issue) doi: 10.1101/gad. 1666108.

Kumar, V., Carlson, J.E., Ohgi, K.A., Edwards, T.A., Rose, D.W. Escalante, C.R., Rosenfeld, M.G., and Aggarwal, A.K. 2002 Transcription corepressor $\mathrm{CtBP}$ is an $\mathrm{NAD}^{+}$-regulated dehydrogenase. Mol. Cell 10: 857-869.

Lagouge, M., Argmann, C., Gerhart-Hines, Z., Meziane, H., Lerin, C., Daussin, F., Messadeq, N., Milne, J., Lambert, P., Elliott, P., et al. 2006. Resveratrol improves mitochondrial function and protects against metabolic disease by activating SIRT1 and PGC-1 $\alpha$. Cell 127: 1109-1122.

Leonardsson, G., Steel, J.H., Christian, M., Pocock, V., Milligan, S., Bell, J., So, P.W., Medina-Gomez, G., Vidal-Puig, A., White, R., et al. 2004. Nuclear receptor corepressor RIP140 regulates fat accumulation. Proc. Natl. Acad. Sci. 101: 84378442.

Li, Z., Bowerman, S., and Heber, D. 2005. Health ramifications of the obesity epidemic. Surg. Clin. North Am. 85: 681-701.

Lin, J., Wu, P.H., Tarr, P.T., Lindenberg, K.S., St-Pierre, J., Zhang, C.Y., Mootha, V.K., Jager, S., Vianna, C.R., Reznick, R.M., et al. 2004. Defects in adaptive energy metabolism with CNS-linked hyperactivity in PGC-1 $\alpha$ null mice. Cell 119: $121-135$

Lin, J., Handschin, C., and Spiegelman, B.M. 2005. Metabolic control through the PGC-1 family of transcription coactivators. Cell Metab. 1: 361-370.

Milne, J.C., Lambert, P.D., Schenk, S., Carney, D.P., Smith, J.J., Gagne, D.J., Jin, L., Boss, O., Perni, R.B., Vu, C.B., et al. 2007. Small molecule activators of SIRT1 as therapeutics for the treatment of type 2 diabetes. Nature 450: 712-716.

Nedergaard, J., Bengtsson, T., and Cannon, B. 2007. Unexpected evidence for active brown adipose tissue in adult humans. Am. J. Physiol. Endocrinol. Metab. 293: E444-E452. doi: 10.1152/ajpendo.00691.2006.

Nyengaard, J.R., Ido, Y., Kilo, C., and Williamson, J.R. 2004. Interactions between hyperglycemia and hypoxia: Implications for diabetic retinopathy. Diabetes 53: 2931-2938.

Ogden, C.L., Carroll, M.D., Curtin, L.R., McDowell, M.A., Tabak, C.J., and Flegal, K.M. 2006. Prevalence of overweight and obesity in the United States, 1999-2004. IAMA 295: 1549-1555.

Parker, M.G., Christian, M., and White, R. 2006. The nuclear receptor co-repressor RIP140 controls the expression of metabolic gene networks. Biochem. Soc. Trans. 34: 1103-1106. 
Powelka, A.M., Seth, A., Virbasius, J.V., Kiskinis, E., Nicoloro, S.M., Guilherme, A., Tang, X., Straubhaar, J., Cherniack, A.D., Parker, M.G., et al. 2006. Suppression of oxidative metabolism and mitochondrial biogenesis by the transcriptional corepressor RIP140 in mouse adipocytes. J. Clin. Invest. 116: $125-136$.

Puigserver, P., Wu, Z., Park, C.W., Graves, R., Wright, M., and Spiegelman, B.M. 1998. A cold-inducible coactivator of nuclear receptors linked to adaptive thermogenesis. Cell 92: 829-839.

Rodgers, J.T., Lerin, C., Haas, W., Gygi, S.P., Spiegelman, B.M., and Puigserver, P. 2005. Nutrient control of glucose homeostasis through a complex of PGC-1 $\alpha$ and SIRT1. Nature 434: 113-118.

Rosen, E.D. and MacDougald, O.A. 2006. Adipocyte differentiation from the inside out. Nat. Rev. Mol. Cell Biol. 7: 885896.

Seale, P., Kajimura, S., Yang, W., Chin, S., Rohas, L.M., Uldry, M., Tavernier, G., Langin, D., and Spiegelman, B.M. 2007. Transcriptional control of brown fat determination by PRDM16. Cell Metab. 6: 38-54.

Semenza, G.L. 2001. HIF-1, $\mathrm{O}_{2}$, and the 3 PHDs: How animal cells signal hypoxia to the nucleus. Cell 107: 1-3.

Semenza, G.L. 2007a. Oxygen-dependent regulation of mitochondrial respiration by hypoxia-inducible factor 1 . Biochem. I. 405: 1-9.

Semenza, G.L. 2007b. Vasculogenesis, angiogenesis, and arteriogenesis: Mechanisms of blood vessel formation and remodeling. J. Cell. Biochem. 102: 840-847.

Steppan, C.M., Bailey, S.T., Bhat, S., Brown, E.J., Banerjee, R.R., Wright, C.M., Patel, H.R., Ahima, R.S., and Lazar, M.A. 2001. The hormone resistin links obesity to diabetes. Nature 409: 307-312.

Timmons, J.A., Wennmalm, K., Larsson, O., Walden, T.B., Lassmann, T., Petrovic, N., Hamilton, D.L., Gimeno, R.E., Wahlestedt, C., Baar, K., et al. 2007. Myogenic gene expression signature establishes that brown and white adipocytes originate from distinct cell lineages. Proc. Natl. Acad. Sci. 104: 4401-4406.

Truong, M.T., Erasmus, J.J., Munden, R.F., Marom, E.M., Sabloff, B.S., Gladish, G.W., Podoloff, D.A., and Macapinlac, H.A. 2004. Focal FDG uptake in mediastinal brown fat mimicking malignancy: A potential pitfall resolved on PET/CT. AJR Am. J. Roentgenol. 183: 1127-1132.

Uldry, M., Yang, W., St-Pierre, J., Lin, J., Seale, P., and Spiegelman, B.M. 2006. Complementary action of the PGC-1 coactivators in mitochondrial biogenesis and brown fat differentiation. Cell Metab. 3: 333-341.

Wilson-Fritch, L., Burkart, A., Bell, G., Mendelson, K., Leszyk, J., Nicoloro, S., Czech, M., and Corvera, S. 2003. Mitochondrial biogenesis and remodeling during adipogenesis and in response to the insulin sensitizer rosiglitazone. Mol. Cell. Biol. 23: 1085-1094.

Wilson-Fritch, L., Nicoloro, S., Chouinard, M., Lazar, M.A., Chui, P.C., Leszyk, J., Straubhaar, J., Czech, M.P., and Corvera, S. 2004. Mitochondrial remodeling in adipose tissue associated with obesity and treatment with rosiglitazone. $J$. Clin. Invest. 114: 1281-1289.

Ye, J., Gao, Z., Yin, J., and He, Q. 2007. Hypoxia is a potential risk factor for chronic inflammation and adiponectin reduction in adipose tissue of ob/ob and dietary obese mice. Am. J. Physiol. Endocrinol. Metab. 293: E1118-E1128. doi: 10.1152/ajpendo.00435.2007.

Zhang, Q., Piston, D.W., and Goodman, R.H. 2002. Regulation of corepressor function by nuclear NADH. Science 295: $1895-1897$.
Zhang, Q., Wang, S.Y., Nottke, A.C., Rocheleau, J.V., Piston, D.W., and Goodman, R.H. 2006. Redox sensor CtBP mediates hypoxia-induced tumor cell migration. Proc. Natl. Acad. Sci. 103: 9029-9033.

Zhang, Q., Wang, S.Y., Fleuriel, C., Leprince, D., Rocheleau, J.V., Piston, D.W., and Goodman, R.H. 2007. Metabolic regulation of SIRT1 transcription via a HIC1:CtBP corepressor complex. Proc. Natl. Acad. Sci. 104: 829-833. 


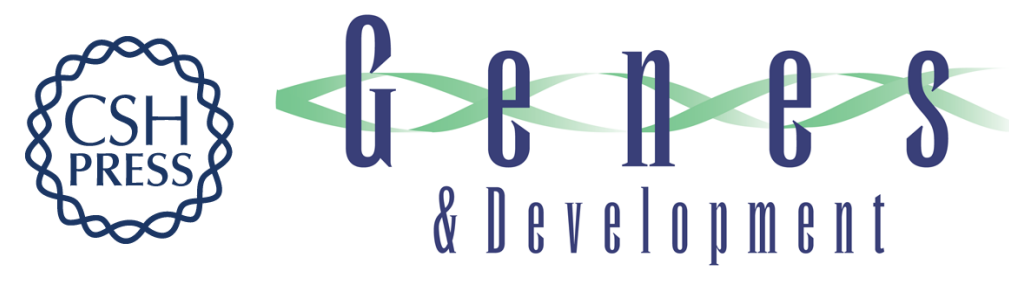

\section{Molecular determinants of brown adipocyte formation and function}

Stephen R. Farmer

Genes Dev. 2008, 22:

Access the most recent version at doi:10.1101/gad.1681308

\section{Related Content}

References

This article cites 44 articles, 13 of which can be accessed free at:

http://genesdev.cshlp.org/content/22/10/1269.full.html\#ref-list-1

Articles cited in:

http://genesdev.cshlp.org/content/22/10/1269.full.html\#related-urls

\section{License}

Email Alerting

Service
Regulation of the brown and white fat gene programs through a PRDM16/CtBP transcriptional complex

Shingo Kajimura, Patrick Seale, Takuya Tomaru, et al.

Genes Dev. May , 2008 22: 1397-1409

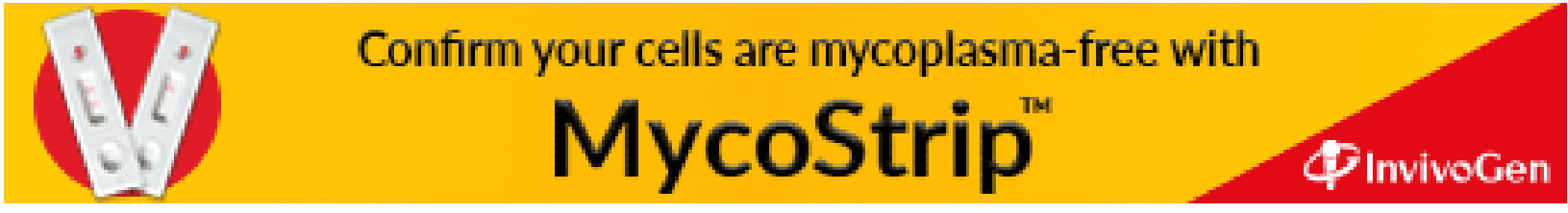

